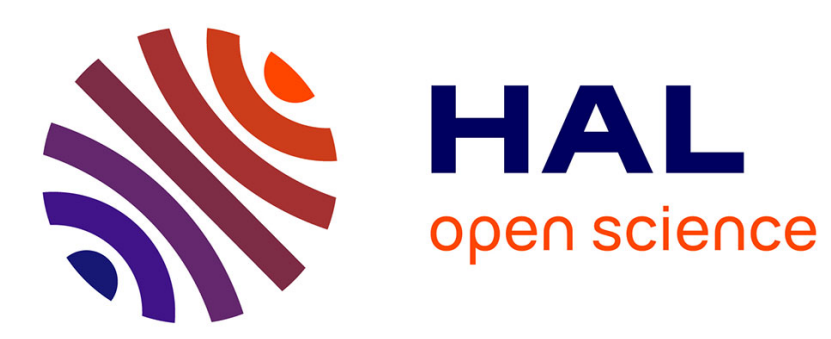

\title{
Functional diagnosability and detectability of nonlinear models based on analytical redundancy relations
}

Nathalie Verdière, Carine Jauberthie, Louise Travé-Massuyès

\section{To cite this version:}

Nathalie Verdière, Carine Jauberthie, Louise Travé-Massuyès. Functional diagnosability and detectability of nonlinear models based on analytical redundancy relations. Journal of Process Control, 2015, 35, pp.1-10. 10.1016/j.jprocont.2015.08.001 . hal-01198408

\section{HAL Id: hal-01198408 \\ https://hal.science/hal-01198408}

Submitted on 15 Sep 2015

HAL is a multi-disciplinary open access archive for the deposit and dissemination of scientific research documents, whether they are published or not. The documents may come from teaching and research institutions in France or abroad, or from public or private research centers.
L'archive ouverte pluridisciplinaire HAL, est destinée au dépôt et à la diffusion de documents scientifiques de niveau recherche, publiés ou non, émanant des établissements d'enseignement et de recherche français ou étrangers, des laboratoires publics ou privés. 


\title{
Functional diagnosability and detectability of nonlinear models based on analytical redundancy relations
}

\author{
Nathalie Verdière ${ }^{1}$, Carine Jauberthie ${ }^{2,3}$, Louise Travé-Massuyès ${ }^{2,4}$ \\ ${ }^{1}$ Normandie Univ, France; ULH, LMAH, F-76600 Le Havre; FR CNRS 3335, ISCN, 25 rue Philippe Lebon 76600 Le Havre, France \\ ${ }^{2}$ CNRS, LAAS, 7 avenue du Colonel Roche, F-31400 Toulouse, France \\ ${ }^{3}$ Université de Toulouse, UPS, LAAS, F-31400 Toulouse, France \\ ${ }^{4}$ Université de Toulouse, LAAS, F-31400 Toulouse, France \\ (Corresponding author: nathalie.verdiere@univ-lehavre.fr).
}

\begin{abstract}
This paper introduces an original definition of diagnosability for nonlinear dynamical models called functional diagnosability. Fault diagnosability characterizes the faults that can be discriminated using the available sensors in a system. The functional diagnosability definition proposed in this paper is based on analytical redundancy relations obtained from differential algebra tools. Contrary to classical definitions, the study of functional diagnosability highlights some of the analytical redundancy relations properties related to the fault acting on the system. Additionally, it gives a criterion for detecting the faults. Interestingly, the proposed diagnosability definition is closely linked to the notion of identifiability, which establishes an unambiguous mapping between the parameters and the output trajectories of a model. This link allows us to provide a sufficient condition for testing functional diagnosability of a system. Numerical simulations attest the relevance of the suggested approach.
\end{abstract}

Keywords: Diagnosability; Identifiability; Nonlinear models; Analytical redundancy relations

\section{Introduction}

Fault diagnosability establishes which faults can be discriminated according to the available sensors in a system. By analyzing diagnosability, it is possible to anticipate the discriminatory power of a diagnoser at run time and to propose solutions to other important problems like the one of selecting the lowest cardinality sensor set that guarantees discriminability of an anticipated set of faults. Diagnosability analysis must be achieved in the framework used to design the diagnoser, which is in our case the model-based framework. The principle of model-based fault diagnosis is to compare the behavior of the system with the predictions that arise from the model and to analyse the sources of discrepancy. In the case of nonlinear models, the classical methods are based on nonlinear observers ([13] for example) and/or Analytical Redundancy Relations (ARRs) ([14], [15]). These latter are relations linking the system inputs, outputs and their derivatives. This paper follows the second track and proposes an extension of the existing definitions and methods for diagnosability and detectability from ARRs. The extension is in line with a gain of dis- criminability.

The considered nonlinear dynamical parametrized models (controlled or uncontrolled) are of the following form:

$$
\left\{\begin{array}{l}
\dot{x}(t, p, f)=g(x(t, p), u(t), f, \varepsilon(t), p), \\
y(t, p, f)=h(x(t, p), u(t), f, \varepsilon(t), p), \\
x\left(t_{0}, p, f\right)=x_{0}, \\
t_{0} \leq t \leq T .
\end{array}\right.
$$

where:

- $x(t, p, f) \in \mathbb{R}^{n}$ and $y(t, p, f) \in \mathbb{R}^{m}$ denote the state variables and the outputs respectively,

- the functions $g$ and $h$ are real, rational and analytic on $M$, where $M$ is an open set of $\mathbb{R}^{n}$ such that $x(t, p, f) \in M$ for every $t \in\left[t_{0}, T\right] . T$ is a finite or infinite time bound,

- $u(t) \in \mathbb{R}^{r}$ is the control vector,

- $f \in \mathbb{R}^{e}$ is the fault vector, 
- $\varepsilon(t)$ is a stochastic vector introducing noise in the system,

- the vector of parameters $p$ belongs to $\mathcal{U}_{\mathcal{P}}$, where $\mathcal{U}_{\mathcal{P}} \subseteq \mathbb{R}^{q}$ is an a priori known set of admissible parameters,

- the initial conditions $x_{0}$ are assumed to belong to a bounded set $X_{0}$, to be independent of $f$ and to be different from an equilibrium point of the system.

$f=0$ means no fault and $\varepsilon=0$ means no noise. In the case of uncontrolled models $u=0$.

From elimination theory, some differential polynomials, also called input-output representations, that may act as ARRs - since they link system inputs, outputs, parameters and their derivatives - can be obtained. In the last decade, algorithms for obtaining such ARRs have been developed and implemented in softwares as Maple [1]. They are based on differential algebra [6] and allow one to eliminate state variables, which are unknown, from the model. ARRs can be used to detect [5], isolate and estimate faults or in other words to achieve fault detection and isolation (FDI) [17]. To do so, a socalled residual is associated to each ARR, and acts as a consistency indicator [14].

In our paper, faults are considered to disturb the system model (1). Interestingly, there is no restriction about the type of faults. They may act multiplicatively changing the value of some parameter already present in the model or as additional parameters. FDI then relies on the assumption that the model parametrization is suitably chosen so that the faults of the system can be detected and isolated. The purpose of diagnosability analysis is to verify such property.

Some definitions of diagnosability based on ARRs have been proposed in the litterature. A classical diagnosability definition stands in comparing fault signatures [15]. Typically, the fault signature of a fault is a Boolean vector referring to a set of residuals and reporting which residuals are sensitive (with a 1) and not sensitive (with a 0) to the fault. According to [15], the model is said diagnosable if for any two faults, their fault signatures are distinct. Then, if two faults act on the same residuals, the model is not diagnosable.

[2] considers that a system is diagnosable if $f$ is algebraically observable with respect to $u$ and $y$. Defining $f_{i}$ as the $i$ th component of the fault vector $f$, it means that each fault component $f_{i}$ can be written as a solution of a polynomial equation in $f_{i}$ and finitely many time derivatives of inputs $u$ and outputs $y$. This definition can be likened to the definition of identifiability proposed in [10]. Indeed, the parameters are defined globally identifiable if the condition above stands for each parameter $p_{i}$. Considering the fault vector as a parameter vector, classical identifiability and diagnosability as proposed by [2] are hence equivalent. The links between the notions of identifiability and diagnosability and the correspondence between faults and parameters have actually been sensed by several authors among which those of [17]. Their work is based on the key paper [10] that presents a method based on the use of input-output representations - from which ARRs can be built - for studying the identifiability of a model. In [10], input-output representations are obtained with the Ritt's algorithm and checking identifiability may require a lot of manipulations of the model equations. As a result, it is often impossible to obtain such input-output representations for complex systems and, if they are obtained, the order of derivatives is so high that the relations cannot be used as ARRs for FDI, thus the limitation of the method proposed by [2] for diagnosability analysis and FDI. Aware of these problems, [17] relaxes the condition required by global identifiability, and allows the input-output representations to involve several parameters/faults. Input-output representations are used to build residuals evaluated thanks to a statistical change detection method. Hence [17] proposes an FDI method for non linear systems but does not consider diagnosability analysis and the problem of providing conditions for faults to be discriminable.

In [3], thanks to the Rosenfeld-Groebner algorithm, which is by far more efficient than the Ritt's algorithm [1], and to a particular elimination order, the authors propose to study the identifiability of the parameters of a model from differential polynomials that may contain more than one parameters. The advantage of these polynomials is that they present a particular form allowing one to provide general conditions to study identifiability. Furthermore, they contain derivatives of lower order than the ones required by [10]. We borrow the idea of [3] for diagnosability analysis and propose a new definition of diagnosability, called functional diagnosability.

This definition is closely related to the classical definition of identifiability. From this link, definitions of fault detectability and discriminability are proposed and a sufficient condition for verifying functional diagnosability is deduced. The method proposed to verify this condition is easy to implement and allows to detect faults whereas traditional methods fail, as illustrated by the example of the water tanks presented in Section 4.2.

The paper is organized as follows. In Section 2, a general method for obtaining specific ARRs is presented. In Section 3, the definition of functional di- 
agnosability is introduced and linked to the notion of identifiability. From this study, a criterion is given for testing functional diagnosability. Section 4 presents two numerical examples and Section 5 discusses the results and concludes the paper.

\section{Obtention of ARRs}

In the following subsections, the expression of ARRs and how to obtain ARRs through variable elimination are presented.

\subsection{ARRs and their decomposition}

In [14], the authors propose to use ARRs for fault detection and isolation in algebraic dynamic systems. An ARR is a relation deduced from the model of the system that links the system inputs and outputs and their derivatives. Provided that derivatives can be estimated, an ARR is hence a testable relation in the sense that it can be evaluated with the measurements and this is why it is useful in the FDI framework.

The following notations borrowed from [14] are used. If $\vartheta$ is a vector, $\bar{\vartheta}^{(k)}$ is the vector whose components are $\vartheta$ and its time derivatives up to order $k, \bar{\vartheta}$ stands for $\vartheta$ and its time derivatives up to some (unspecified) order. Consider the set of ARRs:

$$
w_{i}(\bar{y}, \bar{u}, f, \bar{\varepsilon}, p)=0, i=1, \ldots, m .
$$

They can be decomposed as:

$$
\begin{aligned}
& w_{i}(\bar{y}, \bar{u}, f, \bar{\varepsilon}, p)=w_{d, i}(\bar{y}, \bar{u}, f, p) \\
& -w_{s, i}(\bar{y}, \bar{u}, f, \bar{\varepsilon}, p)=0,
\end{aligned}
$$

where $w_{d, i}(\bar{y}, \bar{u}, f, p)$ is the deterministic part (a polynomial of degree zero in the components of $\bar{\varepsilon}$ ) and $w_{s, i}(\bar{y}, \bar{u}, f, \bar{\varepsilon}, p)$ is the stochastic part (a polynomial of degree at least one in some components of $\bar{\varepsilon}$ ).

In most cases, there is no simple characterization of the residual's stochastic behavior, in particular for established fault detection procedures. [17] provides an FDI method that perfectly exemplifies how stochastic aspects can be managed. However, other papers like [14] propose to base fault detection on the deterministic part of the residual and we also adopt this assumption. With this assumption, $w_{s, i}(\bar{y}, \bar{u}, f, \bar{\varepsilon}, p)=0$ and ARRs can be rewritten:

$$
w_{i}(\bar{y}, \bar{u}, f, \bar{\varepsilon}, p)=w_{d, i}(\bar{y}, \bar{u}, f, p)=0 .
$$

$w_{d, i}(\bar{y}, \bar{u}, f, p)$ can be decomposed as :

$$
w_{d, i}(\bar{y}, \bar{u}, f, p)=w_{0, i}(\bar{y}, \bar{u}, p)-w_{1, i}(\bar{y}, \bar{u}, f, p),
$$

where $w_{0, i}(\bar{y}, \bar{u}, p)$ is a fault-free term and $w_{1, i}(\bar{y}, \bar{u}, f, p)$ is a term that depends on the fault vector. Consequently:

$$
w_{i}(\bar{y}, \bar{u}, f, p)=w_{0, i}(\bar{y}, \bar{u}, p)-w_{1, i}(\bar{y}, \bar{u}, f, p) .
$$

According to (4), the following relation is always true:

$$
w_{0, i}(\bar{y}, \bar{u}, p)=w_{1, i}(\bar{y}, \bar{u}, f, p) .
$$

The residual defined by $\rho=w_{0, i}(\bar{y}, \bar{u}, p)$ which involves only known variables is used for detecting faults. In the absence of faults, $\rho$ is identically zero for any triple $(\bar{y}, \bar{u}, p)$ which satisfies (1) since $w_{1, i}(\bar{y}, \bar{u}, 0, p)=0 . \quad w_{0, i}(\bar{y}, \bar{u}, p)$ is called the residual computation form and $w_{1, i}(\bar{y}, \bar{u}, f, p)$ the residual internal form.

A fault is defined as detectable if $w_{1, i}(\bar{y}, \bar{u}, f, p) \neq 0$ when $f \neq 0$.

In the following section, a method based on the Rosenfeld-Groebner algorithm for obtaining ARRs is presented. The advantage of the adopted method is to give ARRs of a particular form that is interesting with respect to the functional diagnosability definition given in Section 3.1 and the method for testing functional diagnosability presented in Section 3.4.

\subsection{Obtaining specific ARRs through variable elimina- tion}

In denoting $\theta$ the vector $(f, p)^{T}$, the results of [3] can be directly used. Assume that the system (1) can be rewritten as a differential polynomial system composed of polynomial equations and inequalities ${ }^{1}$ :

$$
\left\{\begin{array}{l}
P(\bar{x}, \bar{u}, \theta)=0, \\
Q(\bar{x}, \bar{y}, \theta)=0, \\
R(\bar{x}, \bar{y}, \theta) \neq 0, \\
\dot{\theta}_{i}=0, i=1, \ldots, q+e .
\end{array}\right.
$$

Notice that the polynomials $R$ correspond to denominators in model (1).

The differential ideal generated by (8) is the collection of polynomials obtained from the polynomials of (8) by addition, differentiation in time, and multiplication by any polynomial in $x, u, y, \theta$. Since an infinite number of differential polynomials can be obtained by repeatedly performing the above operations, the differential ideal is an infinite set of differential polynomials.

\footnotetext{
${ }^{1}$ In general a rational system can be rearranged into a polynomial system as described in [11].
} 
Let $\mathcal{I}$ the radical of the differential ideal generated by (8), that is the collection of polynomials some power of which lies in the differential ideal generated by (8) (see [12],[1], [4] for more details). $\mathcal{I}$ endowed with the following ranking which eliminates the state variables:

$$
[\theta] \prec[y, u] \prec[x]
$$

admits a characteristic presentation $\mathcal{C}$ (i.e. a canonical representative of the ideal) which has the following form [1]:

$$
\begin{aligned}
& \left\{\dot{\theta}_{1}, \ldots, \dot{\theta}_{q+e}, w_{1}(\bar{y}, \bar{u}, \theta), \ldots, w_{m}(\bar{y}, \bar{u}, \theta),\right. \\
& \left.Q_{1}(\bar{y}, \bar{u}, \theta, x), \ldots, Q_{n}(\bar{y}, \bar{u}, \theta, x)\right\}
\end{aligned}
$$

where the leader of the polynomial $w_{i}$ is $y_{i}$ for $i=$ $1, \ldots, m$.

Afterwards, we suppose that $\mathcal{C}(\theta)$, the characteristic presentation $\mathcal{C}$ evaluated in the particular value $\theta$ is equal to $\mathcal{C}_{\theta}$, the characteristic presentation obtained with the elimination ranking $[y, u] \prec[x]$ (see [4] or [16] for more details).

$\mathcal{C}(\theta)$ is proved to contain the differential polynomials $w_{1}(\bar{y}, \bar{u}, \theta), \ldots, w_{m}(\bar{y}, \bar{u}, \theta)$ which can be expressed as:

$$
w_{i}(\bar{y}, \bar{u}, \theta)=\tilde{m}_{0, i}(\bar{y}, \bar{u})+\sum_{k=1}^{n_{i}} \tilde{\gamma}_{k}^{i}(\theta) \tilde{m}_{k, i}(\bar{y}, \bar{u})
$$

where $\left(\tilde{\gamma}_{k}^{i}\right)_{1 \leq k \leq n_{i}}$ are rational in $\theta, \tilde{\gamma}_{v}^{i} \neq \tilde{\gamma}_{w}^{i}(v \neq w)$, $\left(\tilde{m}_{k, i}(\bar{y}, \bar{u})\right)_{1 \leq k \leq n_{i}}$ are differential polynomials with respect to $y$ and $\bar{u}$ and $\tilde{m}_{0, i}(\bar{y}, \bar{u}) \not \equiv 0$.

The polynomials $w_{i}$, called the input-output polynomials, can easily be rewritten in the form:

$$
w_{i}(\bar{y}, \bar{u}, f, p)=m_{0, i}(\bar{y}, \bar{u}, p)-\sum_{k=1}^{s_{i}} \gamma_{k}^{i}(f, p) m_{k, i}(\bar{y}, \bar{u})
$$

where the first part of the polynomial, $m_{0, i}(\bar{y}, \bar{u}, p)$, is not identically equal to zero and does not contain components of $f$. Notice that the number of ARRs corresponds to the number of outputs [1].

According to the expression of the residual (6) of section 2.1, one gets $\rho_{i}=w_{0, i}(\bar{y}, \bar{u}, p)=m_{0, i}(\bar{y}, \bar{u}, p)$ and $w_{1, i}(\bar{y}, \bar{u}, f, p)=\sum_{k=1}^{s_{i}} \gamma_{k}^{i}(f, p) m_{k, i}(\bar{y}, \bar{u})$.

\subsection{A definition of diagnosability based on signatures}

Let us consider $f_{j}$, the $j$ th component of the fault vector $f$, and let us denote by $f_{[j]}$ the fault vector with all components equal to zero but $f_{j} . f_{j}$ as well as $f_{[j]}$ refer to a single fault.

Considering that $m$ residuals are available, the theoretical signature of a fault $f_{j}$ can be defined as follows.
Definition 2.1. The fault signature of $f_{j}$ is the m-vector $\operatorname{Sig}\left(f_{j}\right)$ whose ith component is equal to 1 if the ith residual internal form $w_{1, i}(\bar{y}, \bar{u}, f, p)$ contains the fault $f_{j}$ and equal to 0 otherwise.

From this definition, discriminality and diagnosability definitions can be given [15].

Definition 2.2. Two faults $f_{i}$ and $f_{j}$ are discriminable if their signatures are different. When all the faults are discriminable, the model is said diagnosable.

According to the above diagnosability definition, if two faults act on the same residuals, the model is not diagnosable. In the following section, a definition of functional diagnosability is proposed. This definition takes into account the fact that a residual can be impacted by two faults but still have different behaviors depending on the fault.

\section{Functional diagnosability}

\subsection{Definition of functional diagnosability and related concepts}

The functional signature of a fault $f_{j}$ is given by the vector formed of the internal form of the $m$ residuals by considering that only the fault $f_{j}$ is acting on the system. In other words, it is given by the vector whose $i$ th component is $w_{1, i}\left(\bar{y}, \bar{u}, f_{[j]}, p\right)$, that is the polynomial obtained from $w_{1, i}(\bar{y}, \bar{u}, f, p)$ by considering all the components of $f$ equal to zero but the $j$ th component equal to $f_{j}$. Formally, the definition is the following:

Definition 3.1. The functional fault signature is a function F Sig which associates to a fault $f_{j}$, the vector $\left(w_{1, i}\left(\bar{y}, \bar{u}, f_{[j]}, p\right)\right)_{i=1, \ldots, m}$.

From the above definition, it is clear that functional fault signatures account explicitly for the effect of the fault magnitude and the input value. This hence brings more information than standard fault signatures.

Let $F \operatorname{Sig}^{(i)}\left(f_{j}\right)=w_{1, i}\left(\bar{y}, \bar{u}, f_{[j]}, p\right)$ denote the $i$ th component of FSig $\left(f_{j}\right)$.

Example 3.1. Consider the following Bernoulli equation:

$$
\dot{y}(t)=\beta_{1} y(t)+\beta_{2} y(t)^{2}, \text { for } t \in[0,5], y(0)=-1 \text {. }
$$

The solution is $y(t)=\frac{\beta_{1} e^{\beta_{1} t}}{\beta_{2}-\beta_{1}-\beta_{2} e^{\beta_{1} t}}$.

We obtain directly $w_{0,1}(\bar{y}, p)=\dot{y}(t)-\beta_{1} y(t)-\beta_{2} y(t)^{2}$. Assume that some positive single faults $f_{1}$ and $f_{2}$ impact additively the two parameters $\beta_{1}$ and $\beta_{2}$ respectively, then $w_{1,1}(\bar{y}, f, p)=f_{1} y(t, p, f)+f_{2} y(t, p, f)^{2}$ 
where $p=\left(\beta_{1}, \beta_{2}\right)^{T}, f=\left(f_{1}, f_{2}\right)^{T}$, and ${ }^{T}$ denotes the transpose of the considered vector. One can deduce that $\operatorname{Sig}\left(f_{1}\right)=\operatorname{Sig}\left(f_{2}\right)=1$ and FSig $\left(f_{1}\right)=$ $f_{1} y\left(., p, f_{[1]}\right), \quad F \operatorname{Sig}\left(f_{2}\right)=f_{2} y\left(., p, f_{[2]}\right)^{2}$ where $y\left(., p, f_{[i]}\right)$ denotes the output impacted by $f_{i}$ only. The two faults are not discriminable according to the classical fault signatures that are equal. However, their functional signatures advocate that the residuals can be distinguished, as shown in more details in section 4.1.

The following definitions are proposed to link the functional signature to the notions of discriminality and diagnosability. The first one is true for all inputs, whereas the second one is verified only for one input.

Definition 3.2. Two faults $f_{j}$ and $f_{l}$ are input-strongly functionally discriminable if for all input $u$, there exists at least one index $i^{*}$ and a finite time $\left.\left.t_{1} \in\right] t_{0}, T\right]$ such that for all $t \in\left[t_{0}, t_{1}\right], F \operatorname{Sig}^{\left(i^{*}\right)}\left(f_{j}\right) \neq F_{S i g}{ }^{\left(i^{*}\right)}\left(f_{l}\right)$. When all the faults are input-strongly functionally discriminable, the model is said input-strongly functionally diagnosable.

Definition 3.3. Two faults $f_{j}$ and $f_{l}$ are input-weakly functionally discriminable if there exits an input $u$, there exists at least one index $i^{*}$, and a finite time $t_{1} \in$ ]$\left.t_{0}, T\right]$ such that for all $t \in\left[t_{0}, t_{1}\right], F \operatorname{Sig}^{\left(i^{*}\right)}\left(f_{j}\right) \neq$ $F S i g^{\left(i^{*}\right)}\left(f_{l}\right)$. When all the faults are input-weakly functionally discriminable, the model is said inputweakly functionally diagnosable.

When the model is uncontrolled, we have the following definition:

Definition 3.4. Two faults $f_{j}$ and $f_{l}$ are functionally discriminable if there exists at least one index $i^{*}$ and a finite time $\left.\left.t_{1} \in\right] t_{0}, T\right]$ such that for all $t \in\left[t_{0}, t_{1}\right]$, $\operatorname{FSig}^{\left(i^{*}\right)}\left(f_{j}\right) \neq F_{S_{i g}}{ }^{\left(i^{*}\right)}\left(f_{l}\right)$. When all the faults are functionally discriminable, the model is said functionally diagnosable.

Detectability is a particular case of discriminability that requires the fault-free situation to be discriminable from the presence of any of the faults, without requiring the faults to be discriminable. Functional detectability can hence be defined based on functional signatures as follows.

Definition 3.5. The fault $f_{i}$ is functionally detectable if the functional signature FSig $\left(f_{i}\right)$ is not equal to the null vector.

Although $\operatorname{Sig}\left(f_{i}\right) \neq 0$ implies $\operatorname{FSig}\left(f_{i}\right) \neq 0$ and vice-versa, functional signatures bring information about the sensitivity of residuals to faults.
The above definition applies to uncontrolled systems. In the case of controlled systems, the distinction between input-strongly and input-weakly detectable can be done in the same way as in definitions 3.2 and 3.3.

Remark- Functional signatures of faults can be collected in a Functional Signature Matrix whose component $(i, j)$ contains the $i$ th residual for the fault $f_{j}$, that is $w_{1, i}\left(\bar{y}, \bar{u}, f_{[j]}, p\right)$. For illustration purposes, consider the following example.

\section{Example 3.2.}

$$
\left\{\begin{array}{l}
\dot{x}_{1}=\left(p_{1}+f_{1}\right)\left(p_{2}+f_{2}\right) x_{1}^{2}+x_{1} x_{2} \\
\dot{x}_{2}=\left(p_{2}+f_{2}\right)\left(p_{3}+f_{3}\right) x_{2}^{2}+x_{2} x_{3} \\
\dot{x}_{3}=\left(p_{1}+f_{1}\right)\left(p_{3}+f_{3}\right) x_{3}^{2}+x_{1} x_{3} \\
y_{1}=x_{1}, y_{2}=x_{2}, y_{3}=x_{3}
\end{array}\right.
$$

It is easy to verify that:

$$
\begin{aligned}
& w_{0,1}(\bar{y}, \bar{u}, p)=\dot{y}_{1}-y_{1} y_{2}-p_{1} p_{2} y_{1}^{2}, \\
& w_{0,2}(\bar{y}, \bar{u}, p)=\dot{y}_{2}-y_{2} y_{3}-p_{2} p_{3} y_{2}^{2}, \\
& w_{0,3}(\bar{y}, \bar{u}, p)=\dot{y}_{3}-y_{1} y_{3}-p_{1} p_{3} y_{2}^{2} .
\end{aligned}
$$

and:

$$
\begin{aligned}
& w_{1,1}(\bar{y}, \bar{u}, f, p)=\left(p_{1} f_{2}+p_{2} f_{1}+f_{1} f_{2}\right) y_{1}^{2}, \\
& w_{1,2}(\bar{y}, \bar{u}, f, p)=\left(p_{2} f_{3}+p_{3} f_{2}+f_{2} f_{3}\right) y_{2}^{2}, \\
& w_{1,3}(\bar{y}, \bar{u}, f, p)=\left(p_{1} f_{3}+p_{3} f_{1}+f_{1} f_{3}\right) y_{3}^{2} .
\end{aligned}
$$

The functional signature are:

$$
\begin{aligned}
& F \operatorname{Sig}\left(f_{1}\right)=\left(p_{2} f_{1} y_{1}^{2}, 0, p_{3} f_{1} y_{3}^{2}\right)^{T}, \\
& F \operatorname{Sig}\left(f_{2}\right)=\left(p_{1} f_{2} y_{1}^{2}, p_{3} f_{2} y_{2}^{2}, 0\right)^{T}, \\
& F S i g\left(f_{3}\right)=\left(0, p_{2} f_{3} y_{2}^{2}, p_{1} f_{3} y_{3}^{2}\right)^{T} .
\end{aligned}
$$

Diagnosability can be analysed thanks to the following table:

\begin{tabular}{c|ccc} 
& & & \\
$F_{\text {Sig }}(f)$ & $f_{1}$ & $f_{2}$ & $f_{3}$ \\
\hline FSig $^{(1)}(f)$ & $p_{2} f_{1} y_{1}^{2}$ & $p_{1} f_{2} y_{1}^{2}$ & 0 \\
FSig $^{(2)}(f)$ & 0 & $p_{3} f_{2} y_{2}^{2}$ & $p_{2} f_{3} y_{2}^{2}$ \\
FSig $^{(3)}(f)$ & $p_{3} f_{1} y_{3}^{2}$ & 0 & $p_{1} f_{3} y_{3}^{2}$
\end{tabular}

Clearly, for $(j, l) \in\{1,2,3\}^{2}, j \neq l, F \operatorname{Fig}\left(f_{j}\right) \neq$ $F S i g\left(f_{l}\right)$ and the model is functionally diagnosable.The system (14) considered in this example is also diagnosable in the classical sense. Nevertheless, the Functional Signature Matrix advantageously shows the impact of the faults on the residuals.

\subsection{A criterion for testing identifiability from ARRs}

Identifiability establishes an unambiguous mapping between the parameters and the output trajectories. [10] 
has proposed definitions for identifiability in the framework of algebra and one of them is recalled below. This definition corresponds to global identifiability at $p$ for the model (1) in which there are no faults. If the initial conditions are not considered, the solutions may not be unique and some solutions may be degenerated. Thus, the set of outputs corresponding to the set of nondegenerated solutions is denoted $Y(p, u)$.

Definition 3.6. The system (1), considered with unknown initial conditions, is said to be globally identifiable at $p^{*}$ with respect to $\mathcal{D}_{\mathcal{M}} \subseteq \mathcal{U}_{\mathcal{P}}$ if there exists an input signal $u^{*}$ such that $Y\left(p^{*}, u^{*}\right) \neq \emptyset$ and

$$
Y\left(p^{*}, u^{*}\right) \cap Y\left(p, u^{*}\right) \neq \emptyset, p \in \mathcal{D}_{\mathcal{M}} \Rightarrow p^{*}=p .
$$

This definition has been extended to local identifiability at $p^{*}$.

The authors of [10] also give a sufficient condition for global identifiability. By using an appropriate elimination order in the Ritt's algorithm, the system (1) leads to a set of differential polynomials called the characteristic set. If all the components $p_{i}$ of the parameter vector $p$ are solutions of a differential equation of the form $P_{i}(\bar{y}, \bar{u}) p_{i}-Q_{i}(\bar{y}, \bar{u})=0$, then the model is globally identifiable at $p$ for any $p \in \mathcal{D}_{\mathcal{M}}$. In addition, if some of the differential polynomials are of degree $>1$ in $p_{i}$, then the model is locally identifiable. These results can be applied to the fault vector of the system so that classical identifiability implies diagnosability as defined in Definition 2.2 of Section 2.3.

However, in most cases, it is impossible to find such nice differential polynomials even if the model structure is globally identifiable. Furthermore, even if the computation terminates, the obtained differential algebraic equations may contain derivatives of high order that induce numerical difficulties for identifying parameters (or faults). Thus, the aim of the following work is to propose a method with wider applicability based on testing functional diagnosability with polynomials that may contain several faults.

The following proposition provides a necessary and sufficient condition for the identifiability of the faults. It is based on the use of the ARRs obtained in Section 2.2. We remind that these are of the form:

$$
F \operatorname{Sig}\left(f_{j}\right)=\left(\sum_{k=1}^{s_{i}} \gamma_{k}^{i}\left(f_{j}, p\right) m_{k, i}(\bar{y}, \bar{u})\right)_{i=1, \ldots, m}
$$

According to [4], one gets the following proposition:
Proposition 3.1. Assume that there exists an index set $I_{0} \subseteq\{1, \ldots, m\}$ such that for all $i \in I_{0}$, the functional determinants $\triangle w_{1, i}(\bar{y}, \bar{u})=\operatorname{det}\left(m_{k, i}(\bar{y}, \bar{u}), k=\right.$ $\left.1, \ldots, s_{i}\right)$ are not identically equal to zero ${ }^{2}$. The model (1) is globally identifiable with respect to the faults if and only if the function $\phi$ defined by :

$\phi: f=\left(f_{1}, \ldots, f_{p}\right) \mapsto\left(\gamma_{1}^{i}(f), \ldots, \gamma_{s_{i}}^{i}(f)\right)_{i \in I_{0}}$ is injective

Proposition 3.1 highlights a link between the injectivity of the function of faults $\phi($.$) and the identifiability of$ faults. The next step consists in linking identifiability of faults and functional diagnosability.

\subsection{Link between identifiability and functional diag- nosability}

Consider $\left(m_{k, i}\left(\bar{y}\left(., p, f_{[j]}\right), \bar{u}\right)\right)_{k=1, \ldots, n_{i}}$ as the set deduced from $\left(m_{k, i}(\bar{y}, \bar{u})\right)_{k=1, \ldots, n_{i}}$ when the output is evaluated for the single fault $f_{j}$.

Proposition 3.2. Assume that there exists an index set $I_{0} \subseteq\{1, \ldots, m\}$ such that for all $i \in I_{0}$, the functional determinants $\triangle w_{1, i}(\bar{y}, \bar{u})=\operatorname{det}\left(m_{k, i}(\bar{y}, \bar{u}), k=\right.$ $\left.1, \ldots, s_{i}\right)$ are not identically equal to zero. If, with the polynomials $w_{i}(\bar{y}, \bar{u}, f, p), i \in I_{0}$, the model is identifiable with respect to the faults then the model is (inputstrongly) functionally diagnosable. The reciprocal is not true.

From Propositions 3.1 and 3.2, one can deduce that a sufficient condition for functional diagnosability is the injectivity of the function $\phi($.$) . However, it is not a nec-$ essary condition according to the counterexample given in the following Proof.

Proof - Assume that the assumptions of Proposition 3.2 are verified.

\footnotetext{
${ }^{2}$ This assumption consists in verifying the linear independence of the $m_{k, i}(\bar{y}, \bar{u}), k=1, \ldots, s_{i}$, by checking that the functional determinant given by the Wronskian [3]

$\triangle w_{1, i}(\bar{y}, \bar{u})=\left|\left(\begin{array}{ccc}m_{1, i}(\bar{y}, \bar{u}) & \ldots & m_{s_{i}, i}(\bar{y}, \bar{u}) \\ m_{1, i}(\bar{y}, \bar{u})^{(1)} & \ldots & m_{s_{i}, i}(\bar{y}, \bar{u})^{(1)} \\ & \ddots & \\ m_{1, i}(\bar{y}, \bar{u})^{\left(s_{i}-1\right)} & \ldots & m_{s_{i}, i}(\bar{y}, \bar{u})^{\left(s_{i}-1\right)}\end{array}\right)\right|$

is not identically equal to zero. If there exists a time point at which the Wronskian is non-zero, then the monomials are linearly independent [7]. In the framework of differential algebra, this condition consists in verifying that this functional determinant is not in the ideal obtained after eliminating state variables. In practice, it can be checked with the function Belong_To of the package DifferentialAlgebra of Maple 16.
} 
Sufficiency The proof is done by contrapositive. Assume that the model is not (input-strongly) functionally diagnosable. Then, there exist two different faults $f_{1}$ and $f_{2}$, (and an input $u$ ), such that for all indexes $i \in\{1, \ldots, m\}$ and for all $t \in$ $\left[t_{0}, T\right]$, we have $F \operatorname{Sig}^{(i)}\left(f_{1}\right)=\operatorname{FSig}^{(i)}\left(f_{2}\right)$. Denote $y\left(., p, f_{[1]}\right) \quad\left(\operatorname{resp} y\left(., p, f_{[2]}\right)\right)$ the output arising from $f_{1}$ (resp. $f_{2}$ ). One gets, for all $i \in\{1, \ldots, m\}, \quad w_{1, i}\left(\bar{y}\left(., p, f_{[1]}\right), \bar{u}, f_{[1]}, p\right)=$ $w_{1, i}\left(\bar{y}\left(., p, f_{[2]}\right), \bar{u}, f_{[2]}, p\right)$, in particular

$$
\begin{aligned}
& \sum_{k=1}^{s_{i}}\left(\gamma_{k}^{i}\left(f_{1}, p\right) m_{k, i}\left(\bar{y}\left(., p, f_{[1]}\right), \bar{u}\right)\right. \\
& \left.\quad-\gamma_{k}^{i}\left(f_{2}, p\right) m_{k, i}\left(\bar{y}\left(., p, f_{[2]}\right), \bar{u}\right)\right)=0 .
\end{aligned}
$$

Three cases can arise:

1. For all indexes $i_{0} \in I_{0}$, one gets that

$$
\begin{aligned}
& m_{1, i_{0}}\left(\bar{y}\left(., p, f_{[1]}\right), \bar{u}\right), \ldots, m_{s_{i_{0}}, i_{0}}\left(\bar{y}\left(., p, f_{[1]}\right), \bar{u}\right), \\
& m_{1, i_{0}}\left(\bar{y}\left(., p, f_{[2]}\right), \bar{u}\right), \ldots, m_{s_{i_{0}}, i_{0}}\left(\bar{y}\left(., p, f_{[2]}\right), \bar{u}\right)
\end{aligned}
$$

are linearly independent. This assumption implies that for all $k=1, \ldots, s_{i_{0}}, \gamma_{k}^{i_{0}}\left(f_{1}, p\right)=\gamma_{k}^{i_{0}}\left(f_{2}, p\right)$ with $f_{1} \neq f_{2}$. Thus, the function $\phi$ defined in Proposition 3.1 is not injective and the model is not identifiable with respect to the faults.

2. We are not in the first case and there exists an in$\operatorname{dex} i_{0} \in I_{0}$, that is a polynomial, such that some of the $m_{l, i_{0}}\left(\bar{y}\left(., p, f_{[2]}\right), \bar{u}\right), l \in\left\{1, \ldots, s_{i_{0}}\right\}$ depend linearly on $\left\{m_{k, i_{0}}\left(\bar{y}\left(., p, f_{[1]}\right), \bar{u}\right)\right\}_{k=1, \ldots, s_{i_{0}}}$, the others not. For example, suppose that, for $l=1, \ldots, v, v<s_{i_{0}}, m_{l, i_{0}}\left(\bar{y}\left(., p, f_{[2]}\right), \bar{u}\right)=$ $\sum_{k=1}^{s_{i_{0}}} \xi_{k, l} m_{k, i_{0}}\left(\bar{y}\left(., p, f_{[1]}\right), \bar{u}\right)$ and $\gamma_{v+1}^{i_{0}}\left(f_{2}, p\right) \neq$ 0 . By definition of the functional signature, one gets:

$$
\begin{aligned}
& F \operatorname{Sig}^{\left(i_{0}\right)}\left(f_{1}\right)=\sum_{k=1}^{s_{i_{0}}} \gamma_{k}^{i_{0}}\left(f_{1}, p\right) m_{k, i_{0}}\left(\bar{y}\left(., p, f_{[1]}\right), \bar{u}\right) \\
& \text { and } \\
& F \operatorname{Sig}^{\left(i_{0}\right)}\left(f_{2}\right)= \\
& \sum_{k=1}^{s_{i_{0}}}\left(\sum_{j=1}^{v} \gamma_{j}^{i_{0}}\left(f_{2}, p\right) \xi_{k, j}\right) m_{k, i_{0}}\left(\bar{y}\left(., p, f_{[1]}\right), \bar{u}\right)+ \\
& \sum_{k=v+1}^{s_{i_{0}}} \gamma_{k}^{i_{0}}\left(f_{2}, p\right) m_{k, i_{0}}\left(\bar{y}\left(., p, f_{[2]}\right), \bar{u}\right) .
\end{aligned}
$$

It follows from (18):

$$
\begin{aligned}
\sum_{k=1}^{s_{i_{0}}}( & \left.\gamma_{k}^{i_{0}}\left(f_{1}, p\right)-\sum_{j=1}^{v} \gamma_{j}^{i_{0}}\left(f_{2}, p\right) \xi_{k, j}\right) \times \\
& m_{k, i_{0}}\left(\bar{y}\left(., p, f_{[1]}\right), \bar{u}\right) \\
& -\sum_{k=v+1}^{s_{i_{0}}} \gamma_{k}^{i_{0}}\left(f_{2}, p\right) m_{k, i_{0}}\left(\bar{y}\left(., p, f_{[2]}\right), \bar{u}\right)=0
\end{aligned}
$$

According to the linear independence hypothesis, for $k \in\left\{v+1, \ldots, s_{i_{0}}\right\}, \gamma_{k}^{i}\left(f_{2}, p\right)$ is equal to 0 , which is impossible since $\gamma_{v+1}^{i_{0}}\left(f_{2}, p\right)$ is assumed nonzero. Thus, this situation is impossible.

3. Assume now that for all polynomials $w_{i}$, $i \in\{1, \ldots, m\}$, for all $l \in\left\{1, \ldots, s_{i}\right\}$ $m_{l, i}\left(\bar{y}\left(., p, f_{[2]}\right), \bar{u}\right)$ depends linearly on $\left\{m_{k, i}\left(\bar{y}\left(., p, f_{[1]}\right), \bar{u}\right)\right\}_{k=1, \ldots, s_{i}}, \quad$ that is for all $l$ in $\left\{1, \ldots, s_{i}\right\}, m_{l, i}\left(\bar{y}\left(., p, f_{[2]}\right), \bar{u}\right)=$ $\sum_{k=1}^{s_{i}} \xi_{k, l} m_{k, i}\left(\bar{y}\left(., p, f_{[1]}\right), \bar{u}\right)$. The functional signature of $f_{2}$ can be rewritten:

$$
\begin{aligned}
& F \operatorname{Sig}^{(i)}\left(f_{2}\right)= \\
& \sum_{k=1}^{s_{i}}\left(\sum_{j=1}^{s_{i}} \gamma_{j}^{i}\left(f_{2}, p\right) \xi_{k, j}\right) m_{k, i}\left(\bar{y}\left(., p, f_{[1]}\right), \bar{u}\right) .
\end{aligned}
$$

- If, for all $j \in\left\{1, \ldots, s_{i}\right\}, \gamma_{j}^{i}\left(f_{2}, p\right)$ is solution of the system

$$
\forall k \in\left\{1, \ldots, s_{i}\right\}, \sum_{j=1}^{s_{i}} \gamma_{j}^{i}\left(f_{2}, p\right) \xi_{k, j}=\gamma_{k}^{i}\left(f_{1}, p\right),
$$

the signature of $f_{1}$ can be rewritten

$$
\begin{gathered}
\operatorname{FSig}^{(i)}\left(f_{1}\right)=\sum_{k=1}^{s_{i}}\left(\sum_{j=1}^{s_{i}} \gamma_{j}^{i}\left(f_{2}, p\right) \xi_{k, j}\right) \\
\times m_{k, i}\left(\bar{y}\left(., p, f_{[1]}\right), \bar{u}\right)
\end{gathered}
$$

and $\phi\left(f_{1}\right)=\phi\left(f_{2}\right)$, which means that the model is not identifiable.

- If $\gamma_{j}^{i}\left(f_{2}, p\right)$ is not solution of (22), for example, if $\gamma_{1}^{i}\left(f_{1}, p\right) \neq \sum_{j=1}^{s_{i}} \gamma_{j}^{i}\left(f_{2}, p\right) \xi_{1, j}$, Equation (18) gives:

$$
\begin{aligned}
& \left(\gamma_{1}^{i}\left(f_{1}, p\right)-\sum_{j=1}^{s_{i}} \gamma_{j}^{i}\left(f_{2}, p\right) \xi_{1, j}\right) \\
& \quad \times m_{1, i}\left(\bar{y}\left(., p, f_{[1]}\right), \bar{u}\right) \\
& +\sum_{k=2}^{s_{i}}\left(\gamma_{k}^{i}\left(f_{1}, p\right)-\sum_{j=1}^{s_{i}} \gamma_{j}^{i}\left(f_{2}, p\right) \xi_{k, j}\right) \\
& \quad \times m_{k, i}\left(\bar{y}\left(., p, f_{[1]}\right), \bar{u}\right)=0 .
\end{aligned}
$$


According to the assumptions about functional determinants, the functions $m_{k, i}\left(\bar{y}\left(., p, f_{[1]}\right), \bar{u}\right), \quad k=1, \ldots, s_{i}$ are linearly independent and one can deduce that $\gamma_{1}^{i}\left(f_{1}, p\right)=\sum_{j=1}^{s_{i}} \gamma_{j}^{i}\left(f_{2}, p\right) \xi_{1, j}$, which is not possible.

For proving that the reciprocal is not true, consider the simple differential equation $\dot{y}(t, p)=\left(p_{1}-p_{2}\right) y(t)$, $y(0)=1, p=\left(p_{1}, p_{2}\right)^{T}$, whose solution is $y(t, p)=$ $e^{\left(p_{1}-p_{2}\right) t}, t \in \mathbb{R}$. Assume that two positive and additive single faults $f_{1}$ and $f_{2}$ impact the two parameters $p_{1}$ and $p_{2}$ respectively. From the differential equation, one can deduce that $w_{1,1}(\bar{y}, f, p)=\left(f_{1}-f_{2}\right) y(t, p, f)$. This model is not identifiable according to the faults since the function $\phi:\left(f_{1}, f_{2}\right) \mapsto f_{1}-f_{2}$ is not injective. However, one can deduce that $F \operatorname{Sig}\left(f_{1}\right)=f_{1} y\left(t, p, f_{[1]}\right)=$ $f_{1} e^{\left(p_{1}+f_{1}-p_{2}\right) t}>0, F S i g\left(f_{2}\right)=-f_{2} y\left(t, p, f_{[2]}\right)=$ $-f_{2} e^{\left(p_{1}-p_{2}-f_{2}\right) t}<0$. Since the first signature is positive and the second negative, the two trajectories are distinct on $\mathbb{R}$. Thus, this model is not identifiable with respect to the faults but it is functionally diagnosable.

\subsection{Testing functional diagnosability}

Consider the vector of faults $f=\left(f_{i}\right)_{i=1, \ldots, e}$. The steps for testing functional diagnosability are summed up below.

1. Find the differential polynomials

$$
\left(w_{i}(\bar{y}, \bar{u}, f, p)\right)_{i=1, \ldots, m}
$$

by using, for example, the package DifferentialAlgebra of Maple with the elimination order $[f] \prec$ $[y, u] \prec[x]$.

2. Isolate all the $w_{1, i}(\bar{y}, \bar{u}, f, p), i \in I_{0}$, i.e. the parts of the polynomials $w_{i}(\bar{y}, \bar{u}, f, p)$ containing the faults, for which the functional determinant is not identically equal to zero (see footnote 1 of the Proposition 3.1).

3. Construct the function $\phi$.

4. Verify if $\phi$ is injective.

\subsection{Practical detectability and discriminability}

In the fault-free situation, the functional signature is theoretically equal to the null vector. However, the presence of noise generally corrupts the measured signal, hence the following definitions.

Definition 3.7. The fault $f_{i}$ is $\varepsilon$-functionally detectable if there exist $j \in[1, m]$ and a time interval $\left[t_{1}, t_{2}\right] \subseteq$ $\left[t_{0}, T\right]$ such that for all $t \in\left[t_{1}, t_{2}\right], d\left(F \operatorname{Sig}^{(j)}\left(f_{i}\right), 0\right)>$ $\varepsilon$, where $d$ is a distance on $\mathbb{R}$.
The condition of the above definition, based on the threshold $\varepsilon$, guarantees that at least one residual is above the threshold for a significant time interval, allowing to practically detect the fault $f_{i}$. This condition aims at distinguishing a non null residual from model or measurement noise.

Definition 3.8. If two faults $f_{1}$ and $f_{2}$ act on the same residual, i.e. on the same $w_{1, j}(\bar{y}, \bar{u}, f, p)$, they are said $\varepsilon$-functionally discriminable if there exists a time interval $\left[t_{1}, t_{2}\right] \subseteq\left[t_{0}, T\right]$ such that for all $t \in\left[t_{1}, t_{2}\right]$, $d\left(\operatorname{FSig}^{(j)}\left(f_{1}\right), \operatorname{FSig}^{(j)}\left(f_{2}\right)\right)>\varepsilon$.

These definitions can be extended to input-strongly and input-weakly properties.

\section{Illustrative examples}

\subsection{Bernoulli equation}

Consider the Example 3.1 again and the corresponding residual equal to $\rho=w_{0,1}(\bar{y}, p)=\dot{y}(t)-$ $\beta_{1} y(t)-\beta_{2} y(t)^{2}$. As previously, we assume that some positive single faults $f_{1}$ and $f_{2}$ on the interval $[0,1]$ impact the two parameters $\beta_{1}$ and $\beta_{2}$, respectively. Then $w_{1,1}(\bar{y}, f, p)=f_{1} y(t, p, f)+f_{2} y(t, p, f)^{2}$ and $F \operatorname{Sig}\left(f_{1}\right)=f_{1} y\left(., p, f_{[1]}\right), \quad F \operatorname{Fig}\left(f_{2}\right)=$ $f_{2} y\left(., p, f_{[2]}\right)^{2}$ where for $i=1,2, y\left(., p, f_{[i]}\right)$ denotes the output depending only on fault $f_{i}$. The functional determinant is equal to $\Delta w_{1,1}(\bar{y})=\dot{y} y^{2} \not \equiv 0$ and the function $\phi:\left(f_{1}, f_{2}\right) \mapsto\left(f_{1}, f_{2}\right)$ is injective. According to Proposition 3.1, the model is globally identifiable with respect to the faults. From Proposition 3.2, one can deduce that the model is functionally diagnosable on $[0,5]$, the functional signatures being distinct. Indeed, numerically, if $\beta_{1}=1, \beta_{2}=2, y\left(t, p, f_{[1]}\right)$ is negative on $[0,5]$ and clearly, $F \operatorname{Sig}\left(f_{1}\right) \neq F \operatorname{Sig}\left(f_{2}\right)$ on $[0,5]$. Notice however that the model is not diagnosable in the classical sense.

The residual can be used to detect one of the two faults because its behavior is distinct when one or the other fault is acting on the system. Consider the case of a permanent fault appearing at time $t=0.5 \mathrm{~s}$ with an unknown magnitude between 0.1 and 1 and suppose that the output is disturbed by a Gaussian noise so that the relative error has a maximal value of 0.1 . Figure 1 and Figure 2 represents the residual for several scenarios varying the magnitude of $f_{1}$, respectively $f_{2}$, between 0.1 and 1. From Figure 2, the faults are 0.02functionally detectable and 0.02 -functionally diagnosable. 


\subsection{Water tanks}

The second example concerns two coupled water tanks modeled by:

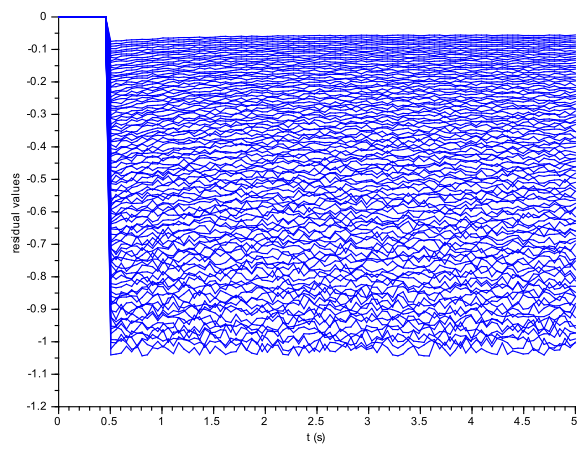

Figure 1: Bernoulli residual when a fault of different magnitude acts on $\beta_{1}$

$$
\left\{\begin{array}{l}
\dot{x}_{1}(t, p)=a_{1} u(t)-a_{2} \sqrt{x_{1}(t, p)}, x_{1}(0)=1 \\
\dot{x}_{2}(t, p)=a_{3} \sqrt{x_{1}(t, p)}-a_{4} \sqrt{x_{2}(t, p)}, x_{2}(0)=0.6 \\
y_{1}(t, p)=a_{5} \sqrt{x_{1}(t, p)} \\
y_{2}(t, p)=a_{6} \sqrt{x_{2}(t, p)}
\end{array}\right.
$$

where $p=\left(a_{i}\right)_{i=1, \ldots, 6}, a_{i} \neq 0$, is the model parameter vector, $x=\left(x_{1}, x_{2}\right)^{T}$ represents the state vector and corresponds to the level in each tank, and $u \neq \equiv$ is the input vector. The water level in the tanks can vary between 0 and 10 .

Let $f_{1}$ denote an unknown additive fault on the actuator signal, $f_{2}$ and $f_{3}$ are additive faults on the two sensors at the output of each of the water tanks, and $f_{4}$ is a clogging fault. $f_{4}=1$ represents a fully clogged pipe and $0<f_{4}<1$ represents partial clogging. In the faulty scenarios, we assume that the faults are introduced at time $t=20 \mathrm{~s}$.

In order to use the Rosenfeld-Groebner algorithm implemented in Maple 16, auxiliary variables $z_{1}(t, p)=$ $\sqrt{x_{1}(t, p)}$ and $z_{2}(t, p)=\sqrt{x_{2}(t, p)}$ are introduced and the model, including the representation of the four faults, is rewritten as:

$$
\left\{\begin{array}{l}
\dot{x}_{1}(t, p)=a_{1}\left(u(t)+f_{1}\right)-a_{2}\left(1-f_{4}\right) z_{1}(t, p), \\
\dot{x}_{2}(t, p)=a_{3}\left(1-f_{4}\right) z_{1}(t)-a_{4} z_{2}(t, p), \\
z_{1}(t, p)^{2}=x_{1}(t, p), \\
z_{2}(t, p)^{2}=x_{2}(t, p), \\
y_{1}(t, p)=a_{5}\left(1-f_{4}\right) z_{1}(t, p)+f_{2}, \\
y_{2}(t, p)=a_{6} z_{2}(t, p)+f_{3}, \\
\dot{f}_{i}=0 .
\end{array}\right.
$$

According to the Rosenfeld-Groebner algorithm, the two ARRs are:

$$
\begin{aligned}
& w_{1}(\bar{y}, \bar{u}, f, p)=w_{0,1}(\bar{y}, \bar{u}, p)-w_{1,1}(\bar{y}, \bar{u}, f, p) \\
& w_{2}(\bar{y}, \bar{u}, f, p)=w_{0,2}(\bar{y}, \bar{u}, p)-w_{1,2}(\bar{y}, \bar{u}, f, p)
\end{aligned}
$$

where

$$
\begin{aligned}
& w_{0,1}=-u a_{1} a_{5}^{2}+\left(a_{2} a_{5}+2 \dot{y}_{1}\right) y_{1}, \\
& w_{1,1}=2 \dot{y}_{1} f_{2}-\left(f_{4}^{2} a_{2} a_{5}-2 f_{4} a_{2} a_{5}\right) y_{1} \\
& -\left(-f_{4}^{2} a_{1} a_{5}^{2}+2 f_{4} a_{1} a_{5}^{2}\right) u \\
& +f_{4}^{2} f_{2} a_{2} a_{5}+f_{4}^{2} f_{1} a_{1} a_{5}^{2}-2 f_{4} f_{2} a_{2} a_{5} \\
& -2 f_{4} f_{1} a_{1} a_{5}^{2}+f_{2} a_{2} a_{5}+f_{1} a_{1} a_{5}^{2}, \\
& w_{0,2}=2 a_{5} \dot{y}_{2} y_{2}-a_{3} a_{6}^{2} y_{1}+a_{4} a_{5} a_{6} y_{2}, \\
& w_{1,2}=2 \dot{y}_{2} f_{3} a_{5}+f_{3} a_{4} a_{5} a_{6}-f_{2} a_{3} a_{6}^{2} .
\end{aligned}
$$


Hence,

$$
\begin{aligned}
F \operatorname{Sig}\left(f_{1}\right)= & \left(-f_{1} a_{1} a_{5}^{2}, 0\right)^{T} \\
\text { FSig }\left(f_{2}\right)= & \left(-2 \dot{y}_{1} f_{2}-f_{2} a_{2} a_{5}, f_{2} a_{3} a_{6}^{2}\right)^{T} \\
\text { FSig }\left(f_{3}\right)= & \left(0,-2 \dot{y}_{2} f_{3} a_{5}-f_{3} a_{4} a_{5} a_{6}\right)^{T} \\
\text { FSig }\left(f_{4}\right)= & \left(y_{1} f_{4}^{2} a_{2} a_{5}-2 y_{1} f_{4} a_{2} a_{5}\right. \\
& \left.\quad-u f_{4}^{2} a_{1} a_{5}^{2}+2 u f_{4} a_{1} a_{5}^{2}, 0\right)^{T} .
\end{aligned}
$$

The functional determinants $\Delta w_{1,1}(\bar{y}, \bar{u})=$ $\operatorname{det}\left(1, u, y_{1}, \dot{y}_{1}\right)=\dot{u}\left(\ddot{y}_{1} y_{1}^{(4)}-y_{1}^{(3)^{2}}\right)-$ $\ddot{u}\left(\dot{y}_{1} y_{1}^{(4)}-y_{1}^{(3)} \ddot{y}_{1}\right)+u^{(3)}\left(\dot{y}_{1} y_{1}^{(3)}-\ddot{y}_{1}^{2}\right)$ and $\Delta w_{1,2}(\bar{y}, \bar{u})=\operatorname{det}\left(\dot{y}_{2}, 1\right)=-\ddot{y}_{2}$ are not identically equal to zero if $u$ is not identically equal to a constant. The function

$$
\begin{aligned}
\phi(f)= & \left(-2 f_{2}, f_{4}^{2} a_{2} a_{5}-2 f_{4} a_{2} a_{5},\right. \\
& -f_{4}^{2} a_{1} a_{5}^{2}+2 f_{4} a_{1} a_{5}^{2}, \\
& -f_{4}^{2} f_{2} a_{2} a_{5}-f_{4}^{2} f_{1} a_{1} a_{5}^{2}+2 f_{4} f_{2} a_{2} a_{5}+ \\
& 2 f_{4} f_{1} a_{1} a_{5}^{2}-f_{2} a_{2} a_{5}-f_{1} a_{1} a_{5}^{2}, \\
& \left.-2 f_{3} a_{5},-f_{3} a_{4} a_{5} a_{6}+f_{2} a_{3} a_{6}^{2}\right)
\end{aligned}
$$

is clearly injective for $\left.f_{4} \in\right] 0,1[$. One can conclude that the model is globally identifiable at $f$ with respect to $] 0,1\left[{ }^{4}\right.$. According to Proposition 3.2 , the model is input-weakly functionally diagnosable. Let us notice that the model is not diagnosable in the classical sense. In the simulations, a simple controller is used to control the water level in the upper tank to follow a square reference signal. The two sensors are disturbed by a Gaussian noise so that the relative error has a maximal value of 0.1 . The parameters of the model are equal to $a_{1}=a_{2}=a_{3}=a_{4}=0.3, a_{5}=a_{6}=1$. Figure 3 shows the water levels in both tanks in a faultfree but noisy simulation. The residuals in their com-

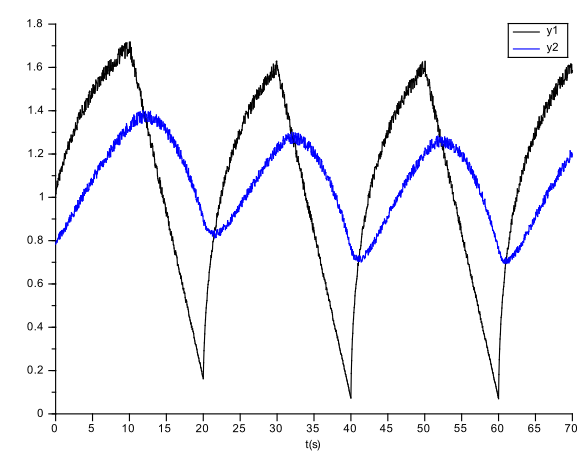

Figure 3: Water level in the upper tank, $y_{1}$, and the lower tank $y_{2}$ during fault-free simulation

putation form $\rho_{1}=-u a_{1} a_{5}^{2}+\left(a_{1} a_{5}+2 \dot{y}_{1}\right) y_{1}$ and $\rho_{2}=2 a_{5} \dot{y}_{2} y_{2}-a_{3} a_{6}^{2} y_{1}+a_{4} a_{5} a_{6} y_{2}$, deduced from (27), are used for detecting the faults. The faults $f_{j}=0.1$, $j \in[1,4]$ are each introduced in turn at time $t=20 \mathrm{~s}$. The derivatives are estimated using an HOSM differentiator ([8], [9]). Figures 4, 5, 6, 7, 8, 9 and 10, 11 represent the residuals $\rho_{1}$ and $\rho_{2}$ when the different faults act on the system.
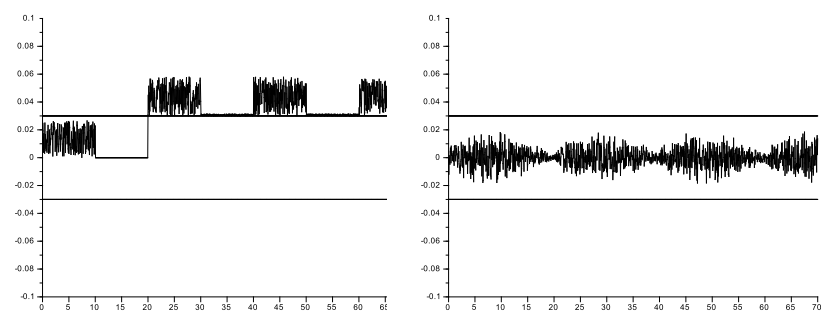

Figure 4: Residual $\rho_{1}$ when fault $f_{1}$ is introduced at time $t=20 s$.

Figure 5: Residual $\rho_{2}$ when fault $f_{1}$ is introduced at time $t=20 s$.
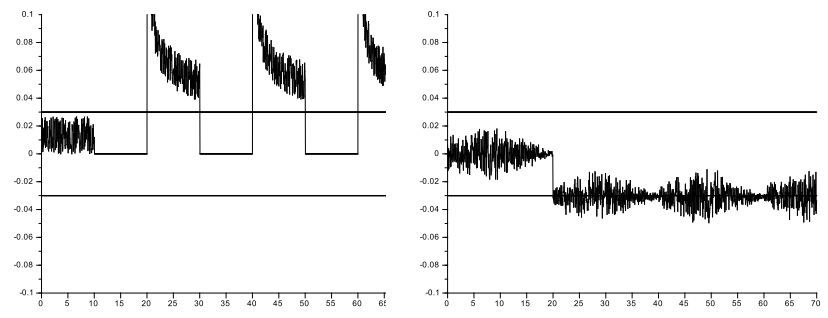

Figure 6: Residual $\rho_{1}$ when fault $f_{2}$ is introduced at time $t=20 s$.

Figure 7: Residual $\rho_{2}$ when fault $f_{2}$ is introduced at time $t=20 s$.

According to the graphs of the residuals, each fault is 0.03-detectable and it is possible to deduce the fault that acts. The ambiguous case is the one for which the faults $f_{1}$ and $f_{4}$ act since they have the same classical signature : the first residual $\rho_{1}$ is not identically equal to zero and the second is zero.

However the behavior graphs are completely different. On the time interval $[21,29]$, for example, they are 0.04 -functionally discriminable. One can also notice that for fault $f_{1}$ the residual $\rho_{1}$ is positive although it is negative for fault $f_{2}$.

\section{Conclusion}

In this paper, a new definition of diagnosability has been proposed as an alternative to classical diagnosabil- 

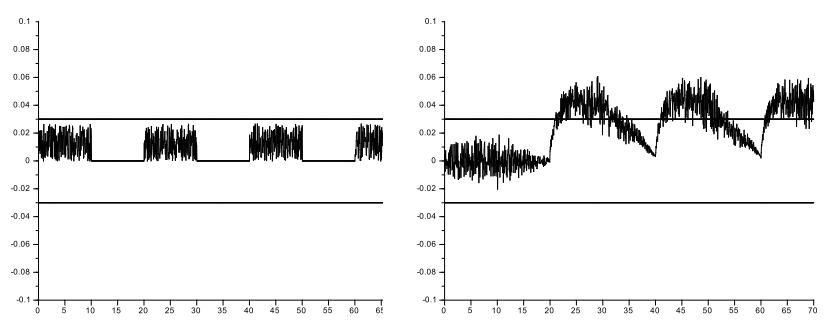

Figure 8: Residual $\rho_{1}$ when fault $f_{3}$ is introduced at time $t=20 s$.

Figure 9: Residual $\rho_{2}$ when fault $f_{3}$ is introduced at time $t=20 s$.
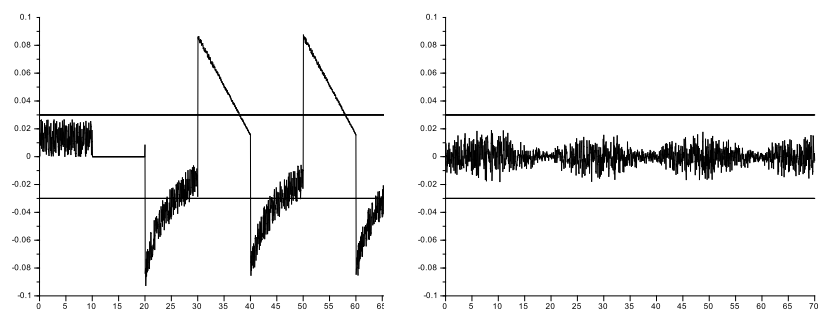

Figure 10: Residual $\rho_{1}$ when fault $f_{4}$ is introduced at time $t=20 s$.

Figure 11: Residual $\rho_{2}$ when fault $f_{4}$ is introduced at time $t=20 s$.

ity in critical cases. This definition is based on analytical redundancy relations and permits to highlight some of their properties according to the fault that acts on the system. It allows us to compare and assess the faulty situation based on the whole trajectories of the residuals. The link between the notion of diagnosability and identifiability which provides a criterion for verifying (functional) diagnosability has been proved. Finally, a method for fault detection has been proposed and the application on two examples highlights the interest of this work. Further investigations, in relation with [5], are planned to extend the presented framework to setmembership models, hence making it possible to nicely consider faults whose value is unknown but bounded.

\section{Acknowledgement}

This work was supported by the French National Research Agency (ANR) in the framework of the project ANR-11-INSE-006 (MAGIC-SPS).

[1] F. Boulier, D. Lazard, F. Ollivier, and M. Petitot. Computing representation for radicals of finitely generated differential ideals. Technical report, Université Lille I, LIFL, 59655, Villeneuve d'Ascq, 1997.
[2] J. C. Cruz-Victoria, R. Martinez-Guerra, and J. J. RinconPasaye. On linear systems diagnosis using differential and algebraic methods. Journal of the Franklin Institute, 345:102-118, 2008.

[3] L. Denis-Vidal, G. Joly-Blanchard, and C Noiret. Some effective approaches to check identifiability of uncontrolled nonlinear systems. Mathematics and Computers in Simulation, 57:35-44, 2001.

[4] L. Denis-Vidal, G. Joly-Blanchard, C. Noiret, and M. Petitot. An algorithm to test identifiability of non-linear systems. In Proceedings of 5th IFAC NOLCOS, St Petersburg, Russia, volume 7, pages 174-178, 2001.

[5] C. Jauberthie, N. Verdière, and L. Travé-Massuyès. Fault detection and identification relying on set-membership identifiability. Annual Reviews in Control, 37:129-136, 2013.

[6] E.R. Kolchin, editor. Differential algebra and algebraic groups. Academic Press, New York, 1973.

[7] M. Krusemeyer. Why does the wronskian work. American Mathematical Monthly, 95:46-49, 1988.

[8] A. Levant. Robust exact differentiation via sliding mode technique. Automatica, 34:379-384, 1998.

[9] A. Levant. Higher-order sliding modes, differentiation and output-feedback control. Int J. Control, 76:924-941, 2003.

[10] L. Ljung and T. Glad. On global identifiability for arbitrary model parametrizations. Automatica, 30:265-276, 1994.

[11] G. Margaria, E Riccomagno, M. Chapell, and H. Wynn. Differential algebra methods for the study of the structural identifiability of rational function state-space models in the biosciences. Mathematical Biosciences, 174:1-26, 2001.

[12] J. Ritt. Differential Algebra. 1st edition, AMS Colloquium, 1950.

[13] R. Seliger and P.M. Frank. Fault diagnosis by disturbance decoupled non-linear obervers. In Proceedings of the 30th IEEE conference on decision and control CDC'91, Brighton, UK, pages 2248-2253, 1991.

[14] M. Staroswiecki and G. Comtet-Varga. Analytical redundancy relations for fault detection and isolation in algebraic dynamic systems. Automatica, 37:687-699, 2001.

[15] L. Travé-Massuyès, T. Escobet, and X. Olive. Diagnosability analysis based on component-supported analytical redundancy relations. IEEE Transactions on Systems, Man and Cybernetics, Part A, 36(6):1146-1160, 2006.

[16] N. Verdière, L. Denis-Vidal, G. Joly-Blanchard, and D. Domurado. Identifiability and estimation of pharmacokinetic parameters of ligands of macrophage mannose receptor. Int. J. Appl. Math. Comput. Sci, 15:101-110, 2005.

[17] Q. Zhang, M. Basseville, and A. Benveniste. Fault detection and isolation in nonlinear dynamic systems: a combined inputoutput and local approach. Automatica, 34(11):1359-1373, 1998.

\section{Vitae}

Nathalie Verdière is Associate Professor at University of Le Havre (France) since 2006. She is a researcher at the Laboratory of Applied Mathematics of Le Havre (http://www.lmah.univ-lehavre.fr) in the "Dynamical Systems" research team (http://www.lmah.univlehavre.fr/systemes_dynamiques). She obtained a Ph.D. degree in 2005 in Applied Mathematics, specialized in system control, from University of Technology 
of Compiègne, France. From 2005 to 2006, she was a temporary Associate Professor at University of Technology of Compiègne. Her research interests concern bifurcations, identifiability analysis and parameter estimation for models described by ordinary or partial differential equations.

Carine Jauberthie is Associate Professor at University Paul Sabatier of Toulouse (France) since 2005. She is a researcher at the CNRS Laboratoire d'Analyse et d'Architecture des Systèmes (LAAS) (http://www.laas.fr) in the Diagnosis and Supervisory Control (DISCO) research team (http://www.laas.fr/DISCO/). She obtained a Ph.D. degree in 2002 in Applied Mathematics, specialized in system control, from University of Technology of Compiègne, France, at ONERA Center of Lille in collaboration with the Laboratory of Applied Mathematics. From 2002 to 2005, she was one year post-doc at Ecole Centrale of Lille (team SyNer) and she taught Mathematics at Ecole des Mines de Douai. Her research interests concern fault detection and diagnosis based on interval analysis, the use of constraint satisfaction approaches, and the analysis of the related properties, including identifiability of linear and nonlinear systems.

Louise Travé-Massuyès is a Research Director of the Centre National de Recherche Scientifique (CNRS), working at Laboratoire d'Analyse et d'Architecture des Systèmes (LAAS) (http://www.laas.fr), Toulouse, France, where she leads the Diagnosis and Supervisory Control (DISCO) research team (http://www.laas.fr/DISCO/). She received a Ph.D. degree in control in 1984 from the Institut National des Sciences Appliquées (INSA); then an Habilitation à Diriger des Recherches from Paul Sabatier University in 1998, all in Toulouse, France. Her main research interests are in Dynamic Systems Supervision and Diagnosis with special focus on Qualitative, Model-Based methods and data mining. She has been particularly active in bridging the AI and Control Model-Based Diagnosis communities. She is a member of the IFAC Safeprocess Technical Committee. 